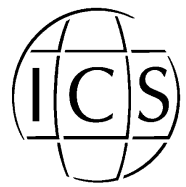

www.ics-elsevier.com

\title{
Influenza pandemic plan: integrated wild bird/ domestic avian/swine/human flu surveillance systems in Taiwan
}

\author{
Chwan-Chuen King ${ }^{\mathrm{a}, *}$, Yi-Jen Liao ${ }^{\mathrm{a}}$, Hui-Lin Yen ${ }^{\mathrm{a}}$, \\ Ming-Chu Cheng ${ }^{b}$, Ching-Ping Tsai ${ }^{c}$, Chuan-Liang Kao ${ }^{a}$, \\ Tsung-Shu Wu ${ }^{\text {a }}$, C.H. Lin ${ }^{d}$, S.C. Chiou ${ }^{d}$, I.J. Su ${ }^{\mathrm{d}}$, \\ N. Cox ${ }^{\mathrm{e}}$, R.G. Webster ${ }^{\mathrm{f}}$ \\ a Institute of Epidemiology, College of Public Health, National Taiwan University, \\ 1 Jen-Ai Road Section 1, Taipei, 100 Taiwan \\ ${ }^{\mathrm{b}}$ Nat. Vet. Res. Inst., Taiwan \\ ${ }^{\mathrm{c}}$ Animal Tech. Inst., Taiwan \\ ${ }^{\mathrm{d}} \mathrm{CDC}$ in Taiwan \\ ${ }^{\mathrm{e}} \mathrm{CDC}$ in Atlanta, GA, USA \\ ${ }^{\mathrm{f}}$ Department Virol. and Molecu. Biol., St. Jude Children's Res. Hosp., Memphis, TN, USA
}

\begin{abstract}
Background: Integrated virological surveillance systems along the transmission chains from animals to humans in areas with large swine/chicken/duck populations and numerous wild migrating birds grasps the frontline timing to detect novel flu viruses. Materials and methods: Animal and human virological surveillance systems have been established to monitor evolutional changes of flu viruses in the same and different hosts/geographical areas over years. Selected sentinel physicians collected specimens from influenza-like patients at local clinics/hospitals in: (a) counties with high populations of swine/avian/wild birds in Taiwan and (b) Kinmen islet — closest to China. Results: Novel animal and human influenza viruses (swine H1N2, avian H5 and H7 and human $\mathrm{H} 3 \mathrm{~N} 2$ within the lineage of A/Fujian/411/2002) were detected in 2002. However, subtypes of H5, H7 and H9 were not found in human isolates in Taiwan till Oct. 2003. Sentinel physician and school absenteeism surveillance systems were more sensitive than human laboratory and respiratory syndromic surveillance systems. Variation patterns of antigenic sites of HA1 in human versus animal flu A viruses were also quite different. Conclusion: The integrated surveillance is very useful in disease control and understanding conditions for the emergence of novel flu viruses with pandemic potential. (C) 2004 Elsevier B.V. All rights reserved.
\end{abstract}

Keywords: Influenza; Integrated surveillance; Taiwan

* Corresponding author. Tel.: +886-2-2341-4371; fax: +886-2-2351-1955.

E-mail address: a1234567@ccms.ntu.edu.tw (C.-C. King). 


\section{Introduction}

Pandemics of influenza A virus in 1918, 1957, and 1968 with more than a million human fatal cases have become the most important global public health problem [1,2]. The emergence of new H5N1 in Hong Kong in 1997 resulted in six fatal cases, the reappearance of H5 at Hong Kong's farms in January and December of 2002 and the recent two human fatal cases traveled from China to Hong Kong in February 2003 plus H7N7 in the Netherlands in 2003 all together imply the urgent need in establishing integrated influenza virologic surveillance system and understanding the conditions to emerge novel influenza $\mathrm{A}$ viruses [2].

Public health officials in Taiwan have to establish the most efficient surveillance systems because Republic of China has not had international health services provided from the World Health Organization (WHO) since 1972. Generally, the animal and human influenza virological surveillance systems in Taiwan are separate and had not communicated frequently as expected until 1998 [3]. Human influenza surveillance systems include sentinel physician surveillance, hospitalization surveillance, physicianbased passive reporting, virological laboratory surveillance and recently established nation-wide school absenteeism surveillance [4]. In animal influenza surveillance, swine, domestic avian and wild bird influenza surveillance systems have been actively implemented since $1998[5,6]$.

The objectives of this study were: (a) to integrate several human flu surveillance systems for early detection of flu epidemics, (b) to establish "integrated animal/human influenza A virologic surveillance systems" in rural areas where many swine farms, avian farms, and habitats of migrating birds are located, (c) to investigate possible virulent influenza A viruses emerging or re-emerging, and (d) to provide solid flu virologic information for better predicting possible pandemic.

\section{Materials and methods}

\subsection{Study design}

\subsubsection{High risk areas}

The same "high risk" areas in Taiwan and Kinmen farms have swine/domestic avian/ bird/human, all four virological surveillance systems to monitor viral changes.

\subsubsection{Epidemiological investigation}

Enthusiastic sentinel physicians collected throat/nasal swabs from: (a) patients manifesting influenza-like illness at local clinics, and district/regional/teaching hospitals located in above study areas, and (b) active virological surveillance by taking more human respiratory specimens when animal flu epidemics occurred.

\subsection{Laboratory diagnosis}

\subsubsection{Avian flu viruses}

Avian flu viruses were isolated by collecting throat and cloacal swabs from live poultry markets in Taipei City/County, local avian farms in northern, central, and southern Taiwan plus isolated Kinmen islet, habitats of migrating birds, water fowl, land-based poultry and 
caged pet birds held in quarantine. The specimens were inoculated into 10-day-old chicken eggs and then allantoic fluids were harvested for typing and subtyping by both reverse transcription-polymerase chain reaction (RT-PCR) with the primers of nucleoprotein (NP), hemagglutinin (HA) and neuraminidase (NA) and serological tests of hemagglutination inhibition (HI), neuraminidase inhibition (NI) [7,8].

\subsubsection{Swine flu viruses}

Swine farms with high density of pigs or close to avian reservoir or avian farms or intense human-animal interactions in Taiwan and Kinmen were selected. In addition, pig herds with outbreaks were also recruited. Nasal swabs of these pigs were collected for virus isolation, typing and subtyping by both serological tests and RT-PCR using universal, M, HA, NA primers [8]. Viral specimens were inoculated into Madin-Darby canine kidney (MDCK) cell line, and grown in 96-well microtiter plates. Immunofluorescence staining used anti-influenza A virus nuclear protein monoclonal antibodies.

\subsubsection{Human flu viruses}

Viral RNA was extracted from the culture supernatants of MDCK cells, and RTPCR was performed by using Uni12 primer (AGCAAAAGCAGG) (WHO manual) for RT and subsequent PCR amplification of cDNA was run by using influenza A specific and subtype-specific primer sets: $M$ gene (52-253, 244 bps), HA gene (1144-R, 650 bps), human H1 (46-633, $650 \mathrm{bps}$ ), and human H3 (365-1072, 785 bps) for typing/ subtyping.

\subsection{Sequence analysis and phylogenetic analysis}

All sequence data were edited and translated by using the DNASTAR. Phylogenetic analysis used Maximum-Parsimony Algorithm and Neighbor-Joining method bootstrapping1000 [9]. The sequences of swine flu H3N2 virus (GenBank accession \#AY377927, \#AY377928) isolated from PingTung of Taiwan and the avian flu H3 viruses [A/Wild Bird/Taiwan/243/02 (H3) and A/Mallard Duck/Taiwan/3.3/03 (H3)] isolated in Taiwan were kindly provided by Ching-Ping Tsai and Ming-Chu Cheng (unpublished data), respectively.

\section{Results}

\subsection{Integration of human influenza surveillance systems}

\subsubsection{Sentinel surveillance}

The improvement of representative sentinel physician surveillance led to detect flu virus activity about 1 month earlier. This surveillance was also much more sensitive than respiratory syndromic surveillance system before and after the SARS epidemic in 2003 (Fig. 1).

\subsubsection{School-based absenteeism surveillance vs. laboratory virological surveillance}

School absenteeism surveillance was more sensitive than laboratory virological surveillance. Moreover, its usefulness is more limited in winter vacation (Fig. 2). 


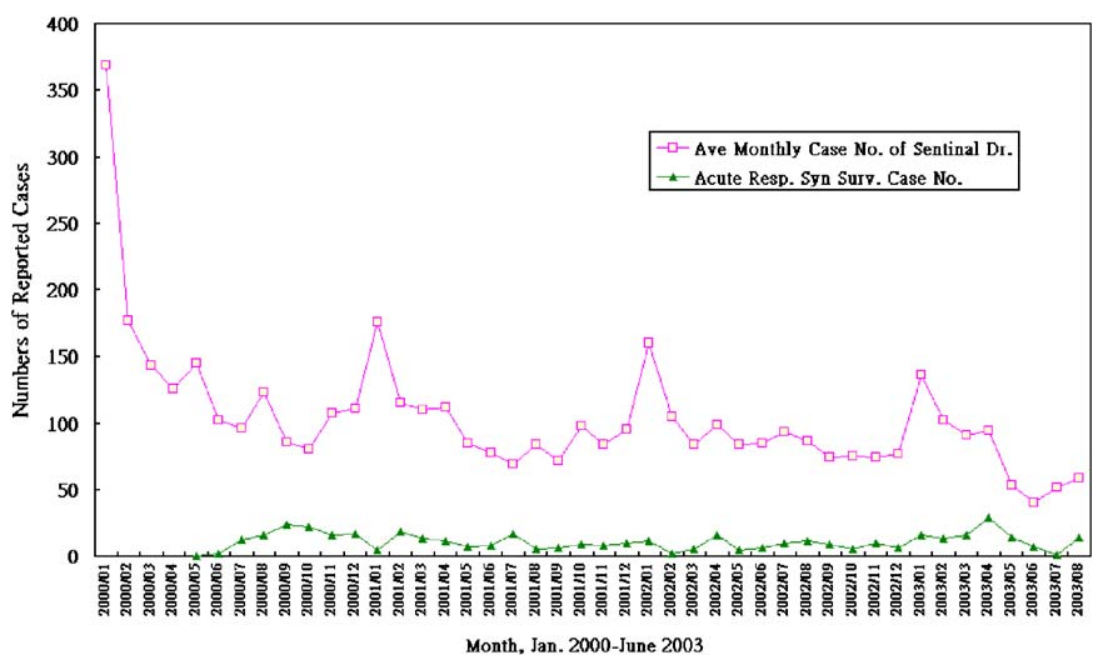

Fig. 1. Monthly comparison of influenza-like illness in sentinel physician-based surveillance vs. acute respiratory syndrome surveillance in Taiwan, 2000-2003.

\subsection{Integration of animal influenza surveillance systems}

Duck flu viruses isolated from the market surveillance had high sequence homology to those coming from migrating birds [11]. In addition, subtypes of swine and bird flu viruses in main Taiwan island were very different from those in Kinmen islet (data not shown).

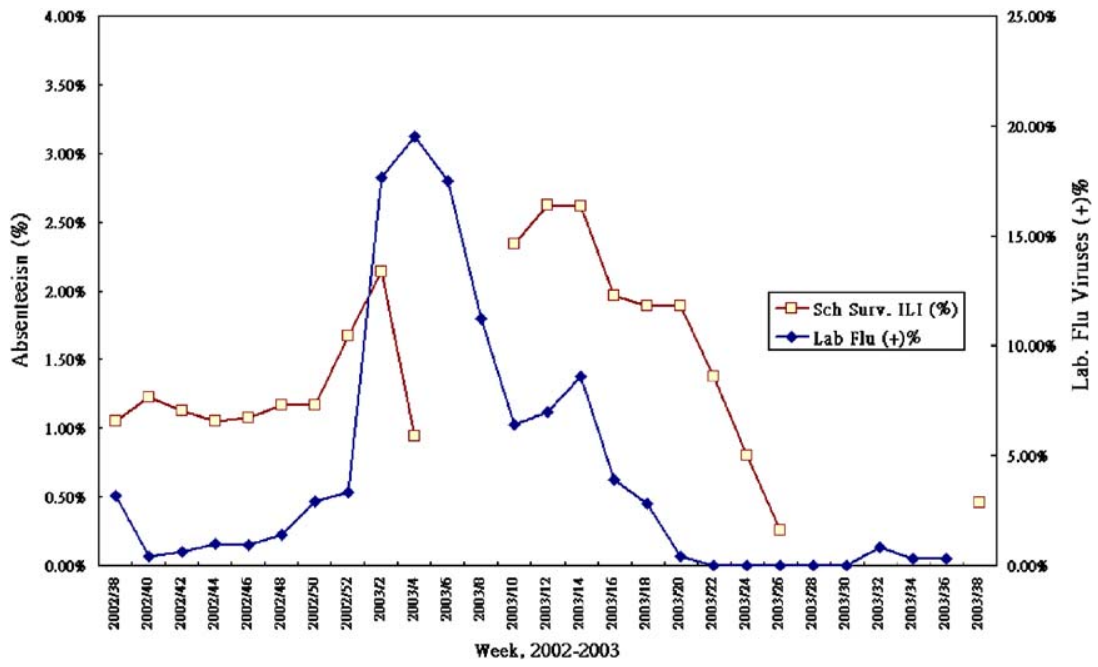

Fig. 2. Weekly comparison of influenza-like illness in school-based absenteeism surveillance vs. laboratory virological surveillance of influenza viruses in Taiwan, 2002-2003. 


\subsection{Integrated animal and human influenza surveillance systems}

Novel human flu viruses such as H5, H7 and H9 had not been detected up to Oct. 2003 even in those farm areas that had outbreaks of novel swine flu H1N2 and avian flu H5 and H7. Furthermore, all human H3N2 viruses did not have animal flu genes in HA, NA and M genes. Many of these human H3N2 isolates in 2002 belong to the lineage of A/Fujian/ $411 / 2002$ (data not shown), indicating that it is very easy to have new influenza viruses transmitted from China to Taiwan.

\subsection{Comparison in amino acid sequences of HA gene}

Three major amino acid sites of HA1 had striking changes between animal and human influenza viruses, including positions of 156, 186, and 225. In addition, one Kinmen human H3N2 isolate obtained from a patient, whose parents are animal handlers and her neighborhood has chicken farms, had avian amino acid D at position 275, compared with amino acid $\mathrm{G}$ for all other human H3N2 isolates.

\section{Discussion}

Most Asian countries had novel influenza viruses isolated frequently before the announcement of the virus strains covered in flu vaccine by the WHO [1,2]. Therefore, it is crucially important to establish molecular epidemiology study team in areas where high virulent influenza viruses may emerge. Since we are in the beginning stage of compiling animal and human influenza viruses together, more and more information will be helpful to understand the connection between ecology and evolution of influenza A viruses [10].

Because specimens need to be taken for virologic surveillance and is very costly, more sensitive community-based sentinel physician and school absenteeism surveillance systems integrated with virological surveillance, once epidemiological conditions exist, will be necessary to detect novel flu virus much earlier in a more systematic and representative approach. Syndromic surveillance is another alternative [12], although it was insensitive in past years in Taiwan. After the epidemic of SARS, timely human respiratory syndromebased virological surveillance covering wider areas has been strengthened to increase its sensitivity in early detection.

\section{References}

[1] N.J. Cox, S.E. Tamblyn, T. Tam, Influenza pandemic planning, Vaccine 21 (16) (2003 May 1) 1801-1803.

[2] R.J. Webby, R.G. Webster, Are we ready for pandemic influenza? Science 302 (5650) (2003 Nov 28) $1519-1522$.

[3] C.-C. King, et al., Integrated seven influenza surveillance systems in Taiwan, in: A.D.M.E. Osterhaus, N. Cox, A.W. Hampson (Eds.), Options for the Control of Influenza IV, Amsterdam, Elsevier, Excerpta Medica, 2001, pp. 107-118.

[4] CDC in Taiwan <www.cdc.gov.tw>.

[5] Annual reports from Taiwan Provincial Research Institute for Animal Health <http://www.library.com.tw/ CUS/L00129.HTM>.

[6] Annual reports from Animal Technology Institute in Taiwan <http://www.atit.org.tw/>.

[7] Y. Guan, et al., H9N2 influenza viruses possessing H5N1-like internal genomes continue to circulate in poultry in southeastern China, J. Virol. 74 (20) (2000) 9372-9380. 
[8] R.G. Webster, The importance of animal influenza for human disease, Vaccine 20 (Suppl. 2) (2002 May 15) S16-S20.

[9] Y. Guan, et al., Emergence of genotypes of H5N1 avian influenza viruses in Hong Kong SAR, Proc. Natl. Acad. Sci. U. S. A. 99 (13) (2002) 8950-8955.

[10] R.G. Webster, The importance of animal influenza for human disease, Vaccine 20 (Suppl. 2) (2002 May 15) S16-S20.

[11] H.L. Yen, et al., Influenza surveillance in poultry market and its inter-spices transmission in Taiwan, in: A. D. M.E. Osterhaus, N. Cox and A. W. Hampson, (Eds.) Options for the Control of Influenza IV, Amsterdam, Elsevier, Excerpta Medica, 2001 pp. 201-211.

[12] J.W. Buehler, et al., Syndromic surveillance and bioterrorism-related epidemics, Emerg. Infect. Dis. 9 (10) (2003 Oct.) 1197-1204. 